\title{
TORUS ACTIONS ON A COHOMOLOGY PRODUCT OF THREE ODD SPHERES
}

\author{
BY \\ CHRISTOPHER ALLDAY(1)
}

\begin{abstract}
The main purpose of this paper is to describe how a torus group may act on a space, $X$, whose rational cohomology ring is isomorphic to that of a product of three odd-dimensional spheres, in such a way that the fixed point set is nonempty, and $X$ is not totally nonhomologous to zero in the associated $X$-bundle, $X_{T} \rightarrow B_{T}$. In the first section of the paper some general results on the cohomology theory of torus actions are established. In the second section the cohomology theory of the above type of action is described; and in the third section the results of the first two sections are used to prove a Golber formula for such actions, which, under certain conditions, bears an interesting interpretation in terms of rational homotopy.
\end{abstract}

Introduction. Equivariant cohomology has proved a useful tool in the theory of transformation groups, especially in the study of torus actions. If $X$ is a $T$-space, $T$ being a torus, then the equivariant cohomology of $X$ is $H^{*}\left(X_{T}\right)$, where $X_{T}$ is the $X$-bundle associated to the universal $T$-bundle. $H^{*}\left(X_{T}\right)$ is usually calculated from the Leray-Serre spectral sequence of the bundle map $X_{T}$ $\rightarrow B_{T}$. The calculation is facilitated when the fixed point set, $X^{T}$, is nonempty, for then the bundle map admits a cross-section; but, even then, the calculation is difficult if $X$ is not totally nonhomologous to zero. Recently Bredon [7] has discovered an action of a torus on a space, $X$, which has the rational cohomology of a product of three odd-dimensional spheres, for which $X^{T}$ is nonempty, but for which $X$ is not totally nonhomologous to zero in $X_{T} \rightarrow B_{T}$ with respect to rational cohomology. This is essentially the simplest known example of such an action on a Poincare duality space. It is the principal purpose of this paper to make a detailed study of such actions, showing that a considerable amount of information can still be obtained.

The paper has three sections. In the first we prove some general results in the cohomology theory of torus actions, including the fact that, if a torus acts on

Received by the editors October 23, 1973.

AMS (MOS) subject classifications (1970). Primary 57E25, 55E99; Secondary 55B25, $55 \mathrm{C} 99$.

Key words and phrases. Torus actions, equivariant cohomology, Golber formula.

(1) This research was supported by NSF Grant No. GP42676. 
a compact Hausdorff space, whose rational cohomology is finite dimensional, then cohomologically speaking there are only finitely many orbit types. (See the proof of Proposition 1.3.) In the second and third sections we specialize to actions of the type described in the first paragraph, above, obtaining a useful splitting theorem (Theorem 2.5) in the second section, which is then applied in the third section to prove a Golber formula for such actions. The Golber formula is the main theorem of the paper, and, although it is not easy to interpret cohomologically, it has a very interesting interpretation in terms of rational homotopy, if the space and fixed point sets in question have the homotopy type of simply-connected CW-complexes. This is particularly suggestive in view of the general lack of knowledge about the homotopy theory of transformation groups.

Throughout the paper, cohomology will be taken to be sheaf-theoretic cohomology with constant rational coefficients and, unless stated otherwise, closed supports. The equivariant cohomology will be that of Borel [3], Hsiang [12] and Quillen [14]. The rational coefficients will be suppressed from our notation: thus, for example, "cohomology dimension" means "rational cohomology dimension," $H^{*}(X)=H^{*}(X ; Q), H_{T}^{*}(X)=H_{T}^{*}(X ; Q)=H^{*}\left(X_{T} ; Q\right)$, and $\operatorname{dim} H^{*}(X)$ means the dimension of $H^{*}(X ; Q)$ as a graded rational vector space.

We make considerable use of the results of Chang and Skjelbred [9]. We recall the following notations and definitions of that paper.

(i) $H^{*}\left(B_{T}\right)=R$.

(ii) For a subtorus $H \subseteq T, P H=\operatorname{ker}\left[R \rightarrow H^{*}\left(B_{H}\right)\right]$.

(iii) If $x \in H_{T}^{*}\left(X^{T}\right)$, then

$$
I_{x}=\left\{a \in R \mid a x \in \operatorname{im}\left[H_{T}^{*}(X) \rightarrow H_{T}^{*}\left(X^{T}\right)\right]\right\} .
$$

(iv) If $M$ is an $R$-submodule of $H_{T}^{*}(X, Y)$, for $Y$ a closed and invariant subspace of $X$, then $\operatorname{ann}^{H}(M)=$ the annihilator in $R$ of the image of $M$ in $H_{T}^{*}\left(X^{H}, Y^{H}\right)$.

(v) $H$ belongs to $M$ if $\operatorname{ann}^{H}(M) \neq(1)$, and if $a \operatorname{ann}^{K}(M)=(1)$, whenever $K \supset H$.

We make note here of the recent generalization of the Borel formula [2].

We would like to thank the referee for some useful suggestions, especially concerning the formulation of Theorem 3.1.

1. Some general results. Let $X$ be a topological space. We shall usually need to assume that $X$ satisfies one or the other of the following conditions.

(A) $X$ is paracompact, the rational cohomology dimension of $X$ is finite, $\operatorname{dim} H^{*}(X)$ is finite; and, if an action of a torus, $T$, on $X$ is given, then the action has finitely many connective orbit types; that is, the set $\theta^{\circ}(X)=\left\{T_{x}^{\circ}\right.$ : 
$x \in X\}$ is finite, where $T_{x}^{\circ}$ is the identity component of the isotropy subgroup of $x$.

(B) $X$ is compact, Hausdorff, and $\operatorname{dim} H^{*}(X)$ is finite. No restrictions on $\theta^{\circ}(X)$ are needed in this case.

Suppose that $X$ is a $T$-space, where $\operatorname{dim}(T)=d$, that $X$ satisfies (A) or (B), and that $Y$ is a closed invariant subspace of $X$, such that $\operatorname{dim} H^{*}(Y)<\infty$. Let $M$ be an $R$-submodule of $H_{T}^{*}(X, Y)$. Then the Poincare series of $M$ in indeterminate $z, P(M, z)$, has the form $\left(1-z^{2}\right)^{-d} p(M, z)$, where $p(M, z)$ is a polynomial [14].

Definition. $\rho_{o}(M)$ is defined to be the order of pole of $P(M, z)$ as $z$ tends to 1 from below. Thus $\rho_{o}(M)=\min \left\{r:\left(1-z^{2}\right)^{r} P(M, z)\right.$ is a polynomial\}.

The following proposition and its corollary have appeared elsewhere, but we include them here with new proofs.

Proposition 1.1 [11]. If $M \neq 0$, then $\rho_{o}(M)=\operatorname{dim}(M)=\max \{\operatorname{rk}(H)$ : $H$ belongs to $M$ \}, where $\operatorname{dim}(M)$ is the dimension of $M$ in the sense of commutative algebra.

Proof. First note that $\mathrm{rk}(H)=\operatorname{coht}(P H)=d-\mathrm{ht}(P H)$ [15]. Now take a composition series for $M,[4$, Chapter $4, \S 3$, No. 1$]$, and observe that the minimal prime ideals appearing in the composition series are the isolated prime ideals of ann $(M)$, and hence have the form $P H$, where $H$ belongs to $M$ [9].

It is clear, then, from the composition series that $\rho_{o}(M)=\max \{\operatorname{rk}(H): H$ belongs to $M\}$. Furthermore, from [15], we have that $\operatorname{dim}(M)=\max \{\operatorname{coht}(P)$ : $P$ is an isolated prime ideal of $\operatorname{ann}(M)\}$.

CoROllaRy $1.2([9],[16])$. If $\operatorname{ann}(M)=\left(a_{1}, \cdots, a_{k}\right) \neq(1)$, then $\rho_{o}(M) \geqslant d-k$.

Proof. Since ann $(M)$ has $k$ generators, if $H$ belongs to $M$, then ht $(P H)$ $\leqslant k[15]$. Thus, $\operatorname{rk}(H) \geqslant d-k$.

For a $T$-space $X$, recall that $S_{k}=\left\{x: \operatorname{corank}\left(T_{x}^{\circ}\right) \leqslant k\right\}$. If $X$ satisfies (A), then $\operatorname{dim} H^{*}\left(S_{k}\right)<\infty$, clearly, since $S_{k}=S_{k-1} \cup \bigcup\left\{X^{H}: \operatorname{corank}(H)\right.$ $=k$ and $\left.H \in \theta^{\circ}(X)\right\}$. The next proposition is useful, in that it saves us from assuming that $\theta^{\circ}(X)$ is finite, so long as $X$ satisfies (B) (cf. [1]).

Proposition 1.3. If $X$ satisfies (B), then $H_{T}^{*}\left(S_{k}\right)$ is finitely generated $R$-module for all $k \geqslant 0$.

Proof. Since $S_{o}=X^{T}$, the result is clear for $k=0$. Suppose that $H_{T}^{*}\left(S_{k}\right)$ is finitely generated, and let $H$ be a subtorus of corank $k+1$. Then 
$X^{H}-S_{k}=X^{H}-S_{k}^{H}$ is open in $S_{k+1}-S_{k}$. Thus $H_{T}^{*}\left(S_{k+1}, S_{k}\right)=$ $\Sigma_{H} H_{T}^{*}\left(X^{H}, S_{k}^{H}\right)$, where $H$ ranges over all subtori of corank $k+1$, and $\Sigma$ denotes direct sum.

By $\left[9\right.$, Lemma 1.1], $H_{T}^{*}\left(X^{H}, S_{k}^{H}\right) \neq 0$ if, and only if, $H_{T}^{*}\left(X^{H}, S_{k}^{H}\right)_{P H} \neq 0$. Thus, by the Localization Theorem,

$$
H_{T}^{*}\left(X^{H}, S_{k}^{H}\right) \neq 0 \Leftrightarrow P H \in \operatorname{Supp}\left(H_{T}^{*}\left(X, S_{k}\right)\right) .
$$

By assumption, $H_{T}^{*}\left(X, S_{k}\right)$ is finitely generated, and so let $P H_{1}, \cdots, P H_{s}$ be the minimal elements of $\operatorname{Supp}\left(H_{T}^{*}\left(X, S_{k}\right)\right)$. Clearly corank $\left(H_{i}\right)>k$, for each $i$, and $H_{T}^{*}\left(X^{H}, S_{k}^{H}\right) \neq 0$ implies that $H \subseteq H_{i}$ for some $i$. Thus, in the direct sum above, $H_{T}^{*}\left(X^{H}, S_{k}^{H}\right)$ is nonzero, only when $H$ has corank $k+1$, and $H=H_{i}$ for some $i$.

Corollary 1.4. If $X$ satisfies (B), then $S_{k}$ satisfies (B) for all $k \geqslant 0$.

Proof. $H_{T}^{*}\left(X^{H}, S_{k}^{H}\right)=0 \Leftrightarrow H^{*}\left(X^{H}, S_{k}^{H}\right)=0$.

REMARK. Cohomology with compact supports was used implicitly in the above proofs, e.g. $H^{*}\left(X^{H}, S_{k}^{H}\right) \cong H_{c}^{*}\left(X^{H}-S_{k}^{H}\right)$.

The following proposition generalizes Proposition 2.4 of [9]

Proposition 1.5. Let $X$ be a T-space satisfying (A) or (B); let $t(X)$ be the torsion submodule of $H_{T}^{*}(X)$. Suppose that

(a) $I_{x}$ is principal, for all homogeneous $x \in H_{T}^{*}\left(X^{T}\right)$;

(b) $t(X)$ is generated by a single element, $y$, say, in $H_{T}^{*}(X)$, and

(c) ann $(t(X))$ is principal.

Then the restriction maps

$$
H_{T}^{*}\left(X, X^{T}\right) \rightarrow H_{T}^{*}\left(S_{1}, X^{T}\right) \text { and } H_{T}^{*}(X) \rightarrow H_{T}^{*}\left(S_{1}\right)
$$

are both monomorphisms.

Proof. Clearly the first monomorphism implies the second, and so we shall prove the first only.

Consider the following diagram:

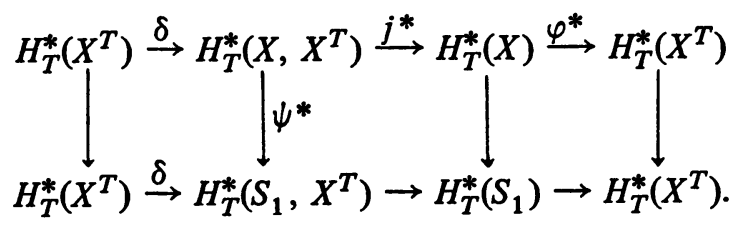

Let $M=\operatorname{ker} \psi^{*}$. We must show that $M=0$.

Let $x \in M$, and suppose that $j^{*}(x)=0$. Then we may lift $x$ to $H_{T}^{*}\left(X^{T}\right)$. We may now mimic the proof of $[9$, Proposition 2.4], which is valid, whether 
there be finitely many corank one isotropy subgroups or not, to show that $x=0$. Thus $j^{*}$ maps $M$ monomorphically into $\operatorname{im} j^{*}=\operatorname{ker} \varphi^{*}$, and $\operatorname{ker} \varphi^{*}=t(X)$, by the Localization Theorem.

Viewing $M$ as a submodule of $t(X)$, suppose that $M$ is generated by $\left\{r_{1} y\right.$, $\left.\cdots, r_{k} y\right\}$, where $r_{i} \in R$, for $1 \leqslant i \leqslant k$. Let ann $(t(X))=(a)$, for $a \in R$, and set $\operatorname{ann}(M)=J$.

Then $b \in J \Longleftrightarrow a \mid b r_{i}$, for each $i$. Let $h_{i}$ be the highest common factor of $a$ and $r_{i}$, so that $a=h_{i} a_{i}$ and $r_{i}=h_{i} r_{i}^{\prime}$, say, where $a_{i}$ and $r_{i}^{\prime}$ are relatively prime in $R$. Let $a^{\prime}$ be the lowest common multiple of $a_{1}, \cdots, a_{k}$, and set $a^{\prime}=a_{i} a_{i}^{\prime}$.

Since $a^{\prime} r_{i}=a_{i} a_{i}^{\prime} h_{i} r_{i}^{\prime}=a a_{i}^{\prime} r_{i}^{\prime}, a^{\prime} \in J$. If $b \in J$, then $a_{i} \mid b r_{i}^{\prime}$ for each $i$, and so $a_{i} \mid b$ for each $i$. Thus $a^{\prime} \mid b$, and so $J=\left(a^{\prime}\right)$.

By Corollary 1.2, then, if $J \neq(1), \rho_{o}(M) \geqslant d-1$ (where $d=\operatorname{dim}(T)$ ). On the other hand, $H_{T}^{*}\left(X, S_{1}\right)$ maps onto $M$, and so $\rho_{o}(M) \leqslant \rho_{o}\left(H_{T}^{*}\left(X, S_{1}\right)\right)$. But we know from [12], or directly from Proposition 1.1, that $\rho_{o}\left(H_{T}^{*}\left(X, S_{1}\right)\right)$ $\leqslant d-2$. Hence $J=(1)$, and so $M=0$.

The next proposition has been proved independently by $T$. Skjelbred in [16]

Proposition 1.6. Let $X$ be a T-space satisfying (A) or (B); let $n=$ $\max \left\{m: H^{m}(X) \neq 0\right\}$. Suppose that $\pi_{2 r}(X) \otimes Q=0$ for $1 \leqslant r \leqslant n / 2$. Then $X^{T}$ is connected.

Proof. If $X$ satisfies (A), there exists a circle $S \subseteq T$ such that $X^{T}=$ $X^{S}$ [11]. The result now follows from Theorem 4.1 of [6].

Suppose that $X$ satisfies (B). From [6], as before, we know that $X^{S}$ is connected for every circle $S \subseteq T$. Suppose that $X^{T}$ is not connected, and let $F_{1}$ and $F_{2}$ be two components of $X^{T}$. Then there exist subtori, $H_{1}, \cdots, H_{s}$, say, such that $F_{1}$ and $F_{2}$ lie in the same component of $X^{H_{i}}$ for each $i$; and if $F_{1}$ and $F_{2}$ lie in the same component of $X^{K}$, for a subtorus $K$, then $K \subseteq H_{i}$ for some $i$ [9]. Choose a circle $S$ such that $\left(S \cap H_{i}\right)^{\circ}=\{e\}$, for each $i$, where $e$ is the identity element of $T$. Then $X^{S}$ is connected, but $S$ is not contained in $H_{i}$, for any $i$, which is a contradiction.

We conclude this section with a result of independent interest, which will not be used in the sequel.

THEOREM 1.7. Let $X$ be a T-space satisfying (A) or (B). Then the following four statements are equivalent.

(i) $X$ is totally nonhomologous to zero, with respect to rational cohomology, in $X_{T} \rightarrow B_{T}$;

(ii) $H_{T}^{*}(X)$ is a free $R$-module; 
(iii) $H_{T}^{*}(X)$ is a projective $R$-module;

(iv) $H_{T}^{*}(X)$ is a flat $R$-module.

Proof. It is well known that (i) $\Rightarrow$ (ii). It is also well known that (ii) $\Leftrightarrow$ (iii) $\Leftrightarrow$ (iv) $\Leftrightarrow \operatorname{Tor}_{1}^{R}\left(H_{T}^{*}(X), Q\right)=0$ ([4], [13]). We need to show that (ii) $\Rightarrow$ (i).

In the rational cohomology Leray-Serre spectral sequence of $X_{T} \rightarrow B_{T}$ give $H_{T}^{*}(X)$ the increasing $R$-module filtration [14, proof of Theorem 2.1], so that $E_{\infty}^{n-t, t} \cong F^{t} H_{T}^{n}(X) / F^{t-1} H_{T}^{n}(X)$. Let

$$
q=\min \left\{j: \exists r \geqslant 2 \text { such that } \operatorname{im} d_{r} \cap E_{r}^{*, j} \neq 0\right\},
$$

assuming that the latter set is nonempty, and hence that (i) is false. We shall assume (ii), and obtain a contradiction.

Let $A=F^{q-1} H_{T}^{*}(X), B=H_{T}^{*}(X)$, and let $i: X \rightarrow X_{T}$ be the inclusion. Then $A$ is a free $R$-module possessing a free basis, $\alpha=\left\{a_{1}, \cdots, a_{r}\right\}$, say, which is mapped by $i *$ to a rational vector space basis for $\sum_{i=0}^{q-1} H^{i}(X)$. If we apply the functor ( ) $\otimes_{R} Q$ to the diagram

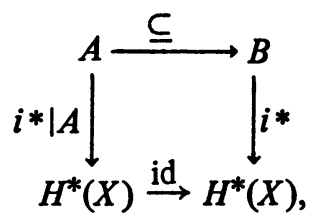

then it is clear that $A \otimes_{R} Q \rightarrow B \otimes_{R} Q$ is injective, and hence $\operatorname{Tor}_{1}^{R}(B / A, Q)$ $\cong \operatorname{Tor}_{1}^{R}(B, Q)$. Since $B$ is free, it follows from [13, Chapter VII, Lemma 6.2], that $B / A$ is free, and so $B \cong A \oplus B / A$. Thus we may extend $\alpha$ to a free $R$ module basis $\alpha^{\prime}=\left\{a_{1}, \cdots, a_{r}, a_{r+1}, \cdots, a_{r+s}\right\}$, say, for $B$, such that $\operatorname{deg}\left(a_{i}\right)$ $<q$ for $i \leqslant r$, and $\operatorname{deg}\left(a_{i}\right) \geqslant q$ for $i>r$.

Let $k=\operatorname{dim} H^{q}(X)$. There exist $b_{1}, \cdots, b_{k}$ in $F^{q} H_{T}^{q}(X)$ such that $\left\{i *\left(b_{1}\right), \cdots, i *\left(b_{k}\right)\right\}$ is a basis for $H^{q}(X)$. From the definition of $q$, there must exist $r_{1}, \cdots, r_{k}$, not all zero, in $R$, such that $\Sigma_{i=1}^{k} r_{i} b_{i} \in F^{q-1} H_{T}^{*}(X)$.

Let $a_{r+1}, \cdots, a_{r+u}$ be the elements of $\alpha^{\prime}$ of degree $q$. Then there exist $t_{i j} \in R$ and $\gamma_{i j} \in Q \subseteq R$ such that

$$
b_{i}=\sum_{j=1}^{r} t_{i j} a_{j}+\sum_{j=1}^{u} \gamma_{i j} a_{r+j}, \text { for } 1 \leqslant i \leqslant k,
$$

and $C=\left(\gamma_{i j}\right)$ is a $k \times u$ matrix of rank $k$ (by applying $i^{*}$ ). It follows that $\Sigma_{i=1}^{k} \gamma_{i j} r_{i}=0$ for $j=1, \cdots, u$. Applying a right inverse of $C$ to this equation, we obtain $r_{i}=0$ for $i=1, \cdots, k$, which is the desired contradiction. 
2. Three odd spheres. For the remainder of this paper, we shall assume that $X$ is a $T$-space satisfying (A) or (B), and that $H^{*}(X)$ is a rational exterior algebra on three generators, $s_{1}, s_{2}$ and $s_{3}$, of degrees $\alpha_{1}, \alpha_{2}$ and $\alpha_{3}$, respectively, each $\alpha_{i}$ being odd. Thus $X$ is a rational Poincaré duality space, and $X \sim S^{\alpha_{1}}$ $\times S^{\alpha_{2}} \times S^{\alpha_{3}}$ over $Q$. We are particularly interested in the case when $X^{T} \neq \varnothing$, but $X$ is not totally nonhomologous to zero in $X_{T} \rightarrow B_{T}$. Such a $T$-space was discovered recently by Bredon [7]. We shall assume that $T$ is acting in such a way, and, hence, we shall assume that $\alpha_{3}>\alpha_{1}+\alpha_{2}$ (and $\alpha_{1} \leqslant \alpha_{2}$ ).

THEOREM 2.1. If $X$ is simple (in the sense of homotopy theory) and homologically locally connected (HLC), then $X^{K}$ is connected for any subtorus $K \subseteq T$; and the Leray-Serre spectral sequence $X_{T} \rightarrow B_{T}$ has only one nonzero differential, which is determined by

$$
d_{r}: E_{r}^{0, \alpha_{3}} \rightarrow E_{r}^{r, \alpha_{1}+\alpha_{2}},
$$

where $r=\alpha_{3}-\left(\alpha_{1}+\alpha_{2}\right)+1$.

Proof. Consider the principal fibration over $X$, induced by the map $X \rightarrow$ $K\left(Q, \alpha_{3}\right)$ given by $s_{3}$. Since $X$ is simple, this fibration is orientable, and it follows that the cohomology of the total space is isomorphic to $\Lambda\left(s_{1}, s_{2}\right)$. Killing $s_{2}$ and $s_{1}$ in like fashion, we deduce that $\pi_{i}(X) \otimes Q \neq 0$, only when $i=\alpha_{1}$, $\alpha_{2}$ or $\alpha_{3}$. Hence the connectedness of each $X^{K}$ follows from Proposition 1.6.

To see the result concerning the differentials in the spectral sequence, take a Moore-Postnikov resolution of $\pi: X_{T} \rightarrow B_{T}$, with respect to rational homotopy. Since $\pi_{i}(X) \otimes Q \neq 0$ only for $i=\alpha_{1}, \alpha_{2}$ and $\alpha_{3}$, the resolution has three stages. Since $X^{T} \neq \varnothing, \pi^{*}$ is injective, and hence the first two stages of the resolution are trivial: the result follows.

REMARK. If, in addition, $X^{T}$ has the homotopy type of a simply-connected CW-complex, then, using a theorem of [6], we can show that $\pi_{n}\left(X^{T}\right) \otimes Q=0$, except when $n$ is odd, that $\operatorname{dim}\left(\pi_{\text {odd }}\left(X^{T}\right) \otimes Q\right)=3$, and that, if $\lambda, \mu$ and $\nu$ generate $\pi_{\text {odd }}\left(X^{T}\right) \otimes Q$, then the only nontrivial Whitehead product of any order in the rational homotopy of $X^{T}$ has the form $\nu=[\lambda, \mu]$. This is the case in Bredon's example.

For the remainder of this paper, we shall assume that the conclusions of Theorem 2.1 obtain (without assuming necessarily that $X$ is simple or HLC), and that $X$ satisfies (B).

DEFINITIONS AND ABBREVIATIONS. (i) "Totally nonhomologous to zero" will be abbreviated "TNHZ."

(ii) A subtorus $H \subseteq T$ will be said to be of type I if $X$ is TNHZ in $X_{H} \rightarrow B_{H}$. $H$ will be said to be of type II otherwise. 
(iii) If $u \in E_{2}^{p, q}$ survives to $E_{r}^{p, q}, E_{n}$ being the Leray-Serre spectral sequence of $X_{T} \rightarrow B_{T}$, then the induced class in $E_{r}^{p, q}$ will be denoted by $[u]_{r}$.

(iv) If $k=\alpha_{3}-\left(\alpha_{1}+\alpha_{2}\right)+1$, then $a \in H^{k}\left(B_{T}\right)$ is given by $d_{k}\left(\left[s_{3}\right]_{k}\right)$ $=\left[a \otimes s_{1} s_{2}\right]_{k}$ in $E_{k}$.

(v) If $H$ is a subtorus of type $\mathrm{I}$, then $H^{*}\left(X^{H}\right)$ is a rational exterior algebra on three odd-degree generators, $s_{1}(H), s_{2}(H)$ and $s_{3}(H)$, say, of degrees $\alpha_{1}(H), \alpha_{2}(H)$ and $\alpha_{3}(H)$, say, respectively [12]. Then, letting $E_{r}(H)$ be the Leray-Serre spectral sequence of $\left(X^{H}\right)_{T} \rightarrow B_{T}$, and, letting $k(H)=\alpha_{3}(H)-$ $\left(\alpha_{1}(H)+\alpha_{2}(H)\right)+1$, we define $a_{H} \in H^{k(H)}\left(B_{T}\right)$, by

$$
d_{k(H)}\left(\left[s_{3}(H)\right]_{k(H)}\right)=\left[a_{H} \otimes s_{1}(H) s_{2}(H)\right]_{k(H)} \text { in } E_{k}(H) .
$$

Note that, from [5] and [8], $X^{T}$ is a rational Poincaré duality space; and from the Localization Theorem, $\operatorname{dim} H^{*}\left(X^{T}\right)=6$. Hence, since

$$
X^{T}=\left(X^{H}\right)^{T}, \quad d_{k(H)}: E_{k(H)}^{*, \alpha_{3}(H)} \rightarrow E_{k(H)}^{*, \alpha_{1}(H)+\alpha_{2}(H)}
$$

is the only nontrivial differential in $E_{r}(H)$.

(vi) If $S$ is a set, then $R S$ will denote the free $R$-module on $S$, and $R\{S\}$ will denote some $R$-module generated by $S$.

(vii) $i: X \rightarrow X_{T}$ and $\varphi:\left(X^{T}\right)_{T} \rightarrow X_{T}$ are the inclusion maps.

Observe that if $H$ is a subtorus of type II, then $\operatorname{dim} H^{*}\left(X^{H}\right)=6$, and $X^{H}$ is TNHZ in $\left(X^{H}\right)_{T} \rightarrow B_{T}$.

Proposition 2.2. There exist elements $x_{1}, x_{2}, x_{13}, x_{23}, x_{123}$ and $y_{12}$ in $H_{T}^{*}(X)$, such that $i *\left(x_{j}\right)=s_{j}(j=1,2), i *\left(x_{j 3}\right)=s_{j} s_{3}(j=1,2), i^{*}\left(x_{123}\right)$ $=s_{1} s_{2} s_{3}$, and $i *\left(y_{12}\right)=s_{1} s_{2}$; and $H_{T}^{*}(X)$ splits as a direct sum of $R$-modules:

$$
H_{T}^{*}(X) \cong R\left\{1, x_{1}, x_{2}, x_{13}, x_{23}, x_{123}\right\} \oplus R\left\{\left\{y_{12}\right\}\right\} .
$$

Furthermore, if $\sigma$ is any cross-section to the bundle $\pi: X_{T} \rightarrow B_{T}$, then these elements may be chosen so that

$$
R\left\{x_{1}, x_{2}, x_{13}, x_{23}, x_{123}\right\} \oplus R\left\{\left\{y_{12}\right\}\right\}=\operatorname{ker} \sigma^{*} .
$$

Proof. Given $H_{T}^{*}(X)$ the increasing $R$-module filtration [14, proof of Theorem 2.1].

Since $X^{T} \neq \varnothing$, a cross-section, $\sigma$, exists. $E_{\infty}$ is a free $R$-module on $E_{\infty}^{0, *}$,

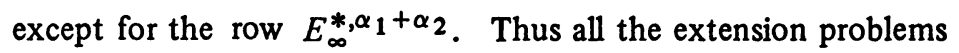

$$
0 \rightarrow F^{t-1} H_{T}^{*}(X) \rightarrow F^{t} H_{T}^{*}(X) \stackrel{p_{t}}{\rightarrow} E^{*-t, t} \rightarrow 0
$$

are trivial except when $t=\alpha_{1}+\alpha_{2}$.

Let $t=\alpha_{1}+\alpha_{2}$, and let $y_{12}^{\prime} \in F^{t} H_{T}^{*}(X)$ be such that $p_{t}\left(y_{12}^{\prime}\right)=$ 
$\left[s_{1} s_{2}\right]_{\infty} .\left[s_{1} s_{2}\right]_{\infty}$ is torsional, with annihilating ideal $(a)$, where $a$ is as defined above. Thus $a y_{12}^{\prime} \in F^{t-1} H_{T}^{*}(X)=R\left\{1, x_{1}, x_{2}\right\}$. Hence, by degrees, $a y_{12}^{\prime}=\pi^{*}(b)$, for some $b \in R$.

Let $y_{12}=y_{12}^{\prime}-\pi * \sigma *\left(y_{12}^{\prime}\right)$; then $p_{t}\left(y_{12}\right)=p_{t}\left(y_{12}^{\prime}\right)$, and $a y_{12}=$ $\pi *(b)-\pi * \sigma * \pi *(b)=0$.

The rest of the proof is straightforward.

REMARKS. (1) im $\pi^{*}$ and ker $\sigma *$ are $R$-subalgebras of $H_{T}^{*}(X)$.

(2) $R\left\{\left\{y_{12}\right\}\right\}$ is the torsion submodule of $H_{T}^{*}(X)$, and its annihilator is $(a)$.

(3) Let $\{1, s, t, u, v, f\}$ be a rational vector space basis for $H^{*}\left(X^{T}\right)$, the basis elements having degrees $0, \sigma, \tau, \nu, \beta, r$, respectively, where $r=f d\left(X^{T}\right)$, and $\sigma$, of course, is not the above cross-section.

Since $X^{T}$ satisfies Poincaré duality, $s, t, u, v$ and $f$ may be chosen so that $\sigma$ and $\tau$ are odd, $\nu$ and $\beta$ are even, and $s v=t u=f$. This notation will be retained throughout the remainder of this paper.

The only other possible nontrivial relations between $s, t, u, v$ and $f$ occur when, say, $\beta=2 \gamma$. Then either $s u=0$ and $u^{2}=0$, or, up to nonzero rational multiples $s u=t$ and $u^{2}=v$. It can be seen easily that these latter relations hold if, and only if, either $x_{13}^{2}$ or $x_{23}^{2}$ is nontorsional.

If $X$ is simple and HLC, and if $X^{T}$ has the homotopy type of a simplyconnected CW-complex, then the rational homotopy of $X^{T}$ is known (see the remark following Theorem 2.1), and we can deduce (from a Postnikov resolution argument) that $\nu=2 \sigma+\tau-1, \beta=\sigma+2 \tau-1$ and $r=2 \sigma+2 \tau-1$. Then $\beta \neq 2 v$, and so $s u=u^{2}=0$, in this case.

COROllaRy 2.3. (a) $\operatorname{ker} \varphi^{*}=R\left\{\left\{y_{12}\right\}\right\}$, and $\varphi * \mid R\left\{1, x_{1}, x_{2}, x_{13}, x_{23}, x_{123}\right\}$ is an isomorphism onto im $\varphi *$.

(b) For any homogeneous $x \in H_{T}^{*}\left(X^{T}\right), I_{x}$ is a principal ideal.

Proof. (a) is immediate from the Localization Theorem, and (b) follows from a straightforward argument (cf. [9, Lemma 2.2]).

Lemma 2.4. Let $R\left\{\left\{y_{12}\right\}\right\}=M$; and let $i_{K}:\left(X^{K}\right)_{T} \rightarrow X_{T}$, and $\varphi_{K}$ : $\left(X^{T}\right)_{T} \rightarrow\left(X^{K}\right)_{T}$ be the inclusions. Let $\operatorname{ker} \varphi_{K}^{*}=M_{K}$, and suppose that $K$ has type I and corank one. Then $\operatorname{ann}\left(M_{K}\right)=\operatorname{ann}\left(i_{K}^{*}(M)\right)$.

Proof. By Proposition 2.2 applied to $X^{K}$, there exists $y_{K 12}$ in $H_{T}^{*}\left(X^{K}\right)$ such that $M_{K}=R\left\{\left\{y_{K_{12}}\right\}\right\}$, and $\operatorname{ann}\left(M_{K}\right)=\left(a_{K}\right)$, where $a_{K}$ is as defined above.

Now there exists $c \in R$, such that $i_{K}^{*}\left(y_{12}\right)=c y_{K 12}$, since $i_{K}^{*}\left(y_{12}\right)$ is torsional.

Thus $b \in \operatorname{ann}\left(i_{K}^{*}(M)\right) \Leftrightarrow a_{K} \mid b c$. 
Since $H_{T}^{*}\left(X^{K}\right) \cong H^{*}\left(B_{K}\right) \otimes H_{T / K}^{*}\left(X^{K}\right)$, it follows that $a_{K}=p_{K}^{m(K)}$, where $p_{K} \in H^{2}\left(B_{T}\right)$ is such that $P K=\left(p_{K}\right)$, and

$$
m(K)=\left(\alpha_{3}(K)-\alpha_{1}(K)-\alpha_{2}(K)+1\right) / 2 .
$$

By the Localization Theorem, $\left(i_{K}\right)_{P K}^{*}$ is an isomorphism, and so there exist $d \in R$ and $r \in R-P K$, such that $i_{K}^{*}\left(d y_{12}\right)=r y_{K 12}$. Hence $a_{K} \mid(d c-r)$, and so $p_{K}+c$. Thus $b \in \operatorname{ann}\left(i_{K}^{*}(M)\right) \Leftrightarrow a_{K} \mid b$.

Note that, in the language of [9], Lemma 2.4 says that $\operatorname{ann}^{K}(M)=\left(a_{K}\right)$. We may now prove the main result of this section.

THEOREM 2.5. (i) a splits as a product of homogeneous linear factors in $R$, one factor for each subtorus, $K$, of type I and corank one, the multiplicity of the factor corresponding to such a $K$ being $\left(\alpha_{3}(K)-\alpha_{1}(K)-\alpha_{2}(K)+1\right) / 2$.

(ii) $\alpha_{3}-\alpha_{1}-\alpha_{2}+1=\Sigma_{K}\left(\alpha_{3}(K)-\alpha_{1}(K)-\alpha_{2}(K)+1\right)$, the sum on the right ranging over all subtori, $K$, of type I and corank one.

Proof. Clearly (i) $\Longrightarrow$ (ii) by taking degrees. With $R\left\{\left\{y_{12}\right\}\right\}=M$, since $\operatorname{ann}(M)=(a)$ is principal, we have from [9], that $\operatorname{ann}(M)=\bigcap_{K} \operatorname{ann}^{K}(M)$, where the intersection runs over all subtori $K$ belonging to $M$, and these have corank one.

By comparing the spectral sequence of $X_{K} \rightarrow B_{K}$ with that of $X_{T} \rightarrow B_{T}$ via inclusion, or by means of a localization argument, it is clear that $a \in P K$ if, and only if, $K$ is of type $I$. Hence the subtori belonging to $M$ are precisely the maximal subtori of type I. The result follows now from Lemma 2.4 .

REMARK. Using Proposition 1.3 and an order of pole argument on $H_{T}^{*}\left(X, S_{1}\right)$, we could have proved (ii) first, and then we could have deduced (i) without reference to Lemma 2.4 .

3. The Golber formula. In this section we shall continue to assume the conditions and notations of $\S 2$. If $H$ is a subtorus of type $I$, then $X^{H}$ has the cohomology of a product of three odd spheres; that is,

$$
H^{*}\left(X^{H}\right)=\wedge\left(s_{1}(H), s_{2}(H), s_{3}(H)\right),
$$

say, where $\operatorname{deg}\left(s_{i}(H)\right)=\alpha_{i}(H)$ is odd, and choosing $\alpha_{i}(H)$ so that $\alpha_{1}(H) \leqslant$ $\alpha_{2}(H) \leqslant \alpha_{3}(H)$, we have that $\alpha_{3}(H)>\alpha_{1}(H)+\alpha_{2}(H)$. If $H$ is a subtorus of type II, then $H^{*}\left(X^{H}\right)=Q\{1, s(H), t(H), u(H), v(H), f(H)\}$, say, where $s(H) v(H)=t(H) u(H)=f(H), \operatorname{deg}(s(H))=\sigma(H), \operatorname{deg}(t(H))=\tau(H), \operatorname{deg}(u(H))$ $=\nu(H), \operatorname{deg}(\nu(H))=\beta(H)$, and $\operatorname{deg}(f(H))=n(H)=f d\left(X^{H}\right)$, since $X^{H}$ satisfies Poincaré duality, with $\operatorname{dim} H^{*}\left(X^{H}\right)=6$. If $H=T$, then $s(H)=s, t(H)$ $=t$, et cetera, and $n(H)=r$. 
DEFinition. (1) If $H$ has type I, set

$e(H)=\left(\alpha_{1}(H)+1\right)\left(\alpha_{2}(H)+1\right)+\left(\alpha_{1}(H)+1\right)\left(\alpha_{3}(H)+1\right)+\left(\alpha_{2}(H)+1\right)\left(\alpha_{3}(H)+1\right)$,

and

$$
\begin{aligned}
g(H)= & n(H)+1+\left(\alpha_{1}(H)+1\right)\left(\alpha_{2}(H)+\alpha_{3}(H)+1\right) \\
& +\left(\alpha_{2}(H)+1\right)\left(\alpha_{1}(H)+\alpha_{3}(H)+1\right) \\
& +\left(\alpha_{3}(H)+1\right)\left(\alpha_{1}(H)+\alpha_{2}(H)+1\right)-\left\{n(H)^{2}+4 n(H)+3\right\} / 4,
\end{aligned}
$$

where $n(H)=\alpha_{1}(H)+\alpha_{2}(H)+\alpha_{3}(H)$.

Thus, $g(H)=2 e(H)-2-\left\{n(H)^{2}+4 n(H)+3\right\} / 4$. If $H=\{e\}, e$ being the identity in $T$, then $X^{H}=X$, and we set $e(H)=e(X), g(H)=g(X), n(H)$ $=n$, and, of course, $\alpha_{i}(H)=\alpha_{i}$.

(2) If $H$ has type II, set

$$
\begin{aligned}
e(H)= & \{(\sigma(H)+1)(\beta(H)+1)+(\tau(H)+1)(\nu(H)+1)\} / 2 \\
& +\left\{n(H)^{2}+8 n(H)+15\right\} / 8,
\end{aligned}
$$

and

$$
g(H)=n(H)+1+(\sigma(H)+1)(\beta(H)+1)+(\tau(H)+1)(\nu(H)+1) .
$$

Thus $g(H)=2 e(H)-2-\left\{n(H)^{2}+4 n(H)+3\right\} / 4$, again.

The following theorem, the Golber formula for this type of torus action, is the main result of this section.

THEOREM 3.1.

(i) $e(X)-e(T)-\sum_{H}[e(H)-e(T)]=\sum_{K}\left[e(K)-e(T)-\sum_{H \supset K}\{e(H)-e(T)\}\right]$.

(ii)

$$
g(X)-g(T)-\sum_{H}[g(H)-g(T)]
$$

$$
=\sum_{K}\left[g(K)-g(T)-\sum_{H \supset K}\{g(H)-g(T)\}\right] .
$$

In both versions of the formula, the sum $\Sigma_{H}$ runs over all subtori, $H$, of corank one, the sum $\Sigma_{K}$ runs over all subtori, $K$, of corank two, and the sums $\Sigma_{H \supset K}$ run over all subtori, $H$, of corank one such that $H \supset K$.

REMARK. If we assume that $X$ and all the fixed point sets $X^{T}, X^{H}, X^{K}$ of relevance to Theorem 3.1 have the homotopy type of simply-connected CWcomplexes, then Theorem 3.1(i) bears a very interesting interpretation in terms of 
rational homotopy. For, if $H$ has type I, then $\alpha_{1}(H), \alpha_{2}(H)$ and $\alpha_{3}(H)$ are the degrees of the generators of the rational homotopy of $X^{H}$; and, if $H$ has type II, then the degrees of the generators of the rational homotopy of $X^{H}$ are

$$
\sigma(H)=\alpha_{1}(H), \quad \text { say, } \quad \tau(H)=\alpha_{2}(H), \text { say, }
$$

and

$$
\sigma(H)+\tau(H)-1=\alpha_{3}(H), \text { say, }
$$

and, once again, we find that

$$
\begin{aligned}
e(H)= & \left(\alpha_{1}(H)+1\right)\left(\alpha_{2}(H)+1\right) \\
& +\left(\alpha_{1}(H)+1\right)\left(\alpha_{3}(H)+1\right)+\left(\alpha_{2}(H)+1\right)\left(\alpha_{3}(H)+1\right) .
\end{aligned}
$$

Thus the same formula serves to define $e(H)$ for either type of subtorus, $H$, when the numbers $\alpha_{i}(H), 1 \leqslant i \leqslant 3$, are taken to be the degrees of the elements of a basis for $\pi_{*}\left(X^{H}\right) \otimes Q$.

The proof of Theorem 3.1, which is an order of pole argument, will occupy the remainder of this section. If $X$ were $\mathrm{TNHZ}$ in $X_{T} \rightarrow B_{T}$, then formula (i) would be the familiar Golber formula ([10] and [9]). Thanks to the results of $\S 1$, the familiar Golber formulae for torus actions on cohomology prociucts of $n$ odd spheres $(n \geqslant 2)$, where the spaces are TNHZ in the associated spectral sequences [9], can be generalized to hold for compact Poincare duality spaces (instead of compact cohomology manifolds).

Lemma 3.2. Let $H_{1}, \cdots, H_{p}$ be the subtori belonging to $f$, and let $K$ be a subtorus of corank two. Then one and only one of the following situations obtains.

(i) There exist $i$ and $j$, with $1 \leqslant i \leqslant j \leqslant p$, such that $K=\left(H_{i} \cap H_{j}\right)^{\circ}$, where ()$^{\circ}$ indicates the taking of the identity component.

(ii) There exist $i$ with $1 \leqslant i \leqslant p$, such that $K \subset H_{i}$ and the restriction $H^{*}\left(X^{K}\right) \rightarrow H^{*}\left(X^{H_{i}}\right)$ is an isomorphism.

(iii) The restriction $H^{*}\left(X^{K}\right) \rightarrow H^{*}\left(X^{T}\right)$ is an isomorphism.

$K$ will be said to be of type (i), (ii), or (iii) accordingly.

Proof. Suppose that (iii) is not the case. Then by [9], there exist $i_{1}$, $\cdots, i_{t}$ such that $1 \leqslant i_{1}<\cdots<i_{t} \leqslant p$, and $K \subset H_{i_{j}}$, for $1 \leqslant j \leqslant t . H_{i_{1}}$, $\cdots, H_{i_{t}}$ are precisely the subtori which belong to $f$ under the action of $T$ on $X^{K}$. If $t \geqslant 2$, then (i) obtains. So suppose that there is only one subtorus which belongs to $f$ under the action of $T$ on $X^{K}$; call it $H_{i}$. By the Borel formula for this action, $n(K)=n\left(H_{i}\right)$, and so (ii) obtains [5]. Clearly (i), (ii) and (iii) are mutually exclusive. 
Definitions. (i) Let $Y$ be a space with $\operatorname{dim} H^{*}(Y)<\infty$. Consider the Poincaré series of $Y, P(Y, z)$. Set

$$
N(Y)=\left.(d / d z) P(Y, z)\right|_{z=1},
$$

and

$$
m(Y)=N(Y)+\left.\left(d^{2} / d z^{2}\right) P(Y, z)\right|_{z=1} .
$$

Abbreviate $m(X)$ by $m, m\left(X^{H}\right)$ by $m(H)$, and $m\left(X^{T}\right)$ by $s$.

(ii) If $H$ is a subtorus of type $I$, then set

$$
c(H)=2\left(\alpha_{1}(H)+\alpha_{2}(H)\right)^{2}-n(H),
$$

and

$$
c=2\left(\alpha_{1}+\alpha_{2}\right)^{2}-n .
$$

In the following lemma, which is the Golber formula in an untidy and disguised form, we make the following summations:

$\Sigma_{H, 1}^{\mathrm{I}}: H$ runs over all subtori of type I, which belong to $f$.

$\Sigma_{H, 1}^{\mathrm{II}}: H$ runs over all subtori of type II, which belong to $f$.

$\Sigma_{K, 2}^{I}: K$ runs over all corank two subtori of types I and (i).

$\Sigma_{K, 2}^{\mathrm{II}}: K$ runs over all corank two subtori of types II and (i). tain $K$.

$\Sigma_{H \supset K}^{\mathrm{I}}: H$ runs over all subtori of type $\mathrm{I}$, which belong to $f$, and which con-

$\Sigma_{H \supset K}^{\mathrm{II}}: H$ runs over all subtori of type II, which belong to $f$, and which contain $K$.

LEMMA 3.3.

$$
\begin{aligned}
m-s & -c-\sum_{H, 1}^{\mathrm{I}}(m(H)-s-c(H))-\sum_{H, 1}^{\mathrm{II}}(m(H)-s) \\
= & \sum_{K, 2}^{\mathrm{I}}\left[m(K)-s-c(K)-\sum_{H \supset K}^{\mathrm{I}}(m(H)-s-c(H))-\sum_{H \supset K}^{\mathrm{II}}(m(H)-s)\right] \\
& +\sum_{K, 2}^{\mathrm{II}}\left[m(K)-s-\sum_{H \supset K}^{\mathrm{II}}(m(H)-s)\right] .
\end{aligned}
$$

Proof. For a pair of spaces $(A, B)$, we shall write the Poincare series of $H^{*}(A, B)$ in $z$ as $P(A, B)$. If $A$ is a $T$-space, and $B$ is invariant, we shall write the Poincare series of $H_{T}^{*}(A, B)$ in $z$ as $P_{T}(A, B)$. The proof will be done in several steps. Let $\operatorname{dim}(T)=d$.

$$
\lim _{z \rightarrow 1_{-}}\left(1-z^{2}\right)^{d-2} P_{T}\left(X, S_{1}\right)=\lim _{z \rightarrow 1_{-}}\left(1-z^{2}\right)^{d-2} P_{T}\left(S_{2}, S_{1}\right) .
$$


This may be found in [10] or [12], and is an easy consequence of $\S 1$ and the exact sequence in equivariant cohomology of $\left(X, S_{2}, S_{1}\right)$.

$$
\lim _{z \rightarrow 1-}\left(1-z^{2}\right)^{d-2} P_{T}\left(S_{2}, S_{1}\right)=\sum_{K} \lim _{z \rightarrow 1-}\left(1-z^{2}\right)^{d-2} P_{T}\left(X^{K}, S_{1}^{K}\right),
$$

where $K$ runs over all corank two subtori, which belong to $H_{T}^{*}\left(X, S_{1}\right)$. (Proof of Proposition 1.3.)

(3) If $K$ has corank two and type (ii), then $H^{*}\left(S_{1}^{K}, X^{T}\right) \cong H^{*}\left(X^{H_{i}}, X^{T}\right)$, where $H_{i}$ is the subtorus belonging to $f$, which contains $K$. Thus $H^{*}\left(X^{K}, S_{1}^{K}\right)=0$.

If $K$ has corank two and type (iii), then $H^{*}\left(S_{1}^{K}, X^{T}\right)=0$, and again $H^{*}\left(X^{K}, S_{1}^{K}\right)=0$.

Hence, combining (1) and (2), we have that

$$
\lim _{z \rightarrow 1-}\left(1-z^{2}\right)^{d-2} P_{T}\left(X, S_{1}\right)=\sum_{K} \lim _{z \rightarrow 1^{-}}\left(1-z^{2}\right)^{d-2} P_{T}\left(X^{K}, S_{1}^{K}\right),
$$

where $K$ runs over all corank two subtori of type (i).

$$
P_{T}\left(X^{K}, S_{1}^{K}\right)=z\left[P_{T}\left(S_{1}^{K}, X^{T}\right)-P_{T}\left(X^{K}, X^{T}\right)\right] .
$$

This follows from Proposition 1.5.

$$
P_{T}\left(S_{1}^{K}, X^{T}\right)=\sum_{H \supset K} P_{T}\left(X^{H}, X^{T}\right),
$$

where $H$ runs over the subtori which belong to $f$, and which contain $K$.

The proof now depends upon the calculation of $\lim _{z \rightarrow 1-}\left(1-z^{2}\right)^{d-2} P_{T}\left(X^{L}, X^{T}\right)$ for various subtori, $L$, of corank one or two. These calculations are straightforward, when $L$ has type II, and almost equally easy when $L$ has type I, by Proposition 2.2. The formula thus obtained can be simplified using the Borel formula and Theorem 2.5(ii), and the lemma follows.

Proof of THEOREM 3.1. If $L$ has type $I$, then

Therefore,

$$
\begin{aligned}
m(L)= & \alpha_{1}(L)^{2}+\alpha_{2}(L)^{2}+\alpha_{3}(L)^{2}+\left(\alpha_{1}(L)+\alpha_{2}(L)\right)^{2} \\
& +\left(\alpha_{1}(L)+\alpha_{3}(L)\right)^{2}+\left(\alpha_{2}(L)+\alpha_{3}(L)\right)^{2}+n(L)^{2} .
\end{aligned}
$$

$$
\begin{aligned}
m(L)-c(L)= & -4 e(L)+7 n(L)^{2} / 2+8 n(L)+23 / 2-k(L)^{2} / 2 \\
& +n(L) k(L)+k(L),
\end{aligned}
$$

where $k(L)=\alpha_{3}(L)-\alpha_{1}(L)-\alpha_{2}(L)+1$.

$$
\begin{aligned}
s & =\sigma^{2}+\tau^{2}+\nu^{2}+\beta^{2}+r^{2} \\
& =-2((\sigma+1)(\beta+1)+(\tau+1)(\nu+1))+3 r^{2}+4 r+4 \\
& =-4 e(T)+7 r^{2} / 2+8 r+23 / 2 .
\end{aligned}
$$


Therefore,

$$
\begin{aligned}
m(L)-s-c(L)= & -4\{e(L)-e(T)\}+7\left\{n(L)^{2}-r^{2}\right\} / 2 \\
& +8\{n(L)-r\}-k(L)^{2} / 2+n(L) k(L)+k(L) .
\end{aligned}
$$

If $L$ has type II, then

$$
m(L)-s=-4\{e(L)-e(T)\}+7\left\{n(L)^{2}-r^{2}\right\} / 2+8\{n(L)-r\} .
$$

By the Borel formula and Theorem 2.5(ii), it remains only to establish the following equalities.

(1)

$$
\begin{aligned}
n^{2} & -r^{2}-\sum_{H, 1}^{\mathrm{I}}\left(n(H)^{2}-r^{2}\right)-\sum_{H, 1}^{\mathrm{II}}\left(n(H)^{2}-r^{2}\right) \\
= & \sum_{K, 2}^{1}\left[n(K)^{2}-r^{2}-\sum_{H \supset K}^{\mathrm{I}}\left(n(H)^{2}-r^{2}\right)-\sum_{H \supset K}^{\mathrm{II}}\left(n(H)^{2}-r^{2}\right)\right] \\
& +\sum_{K, 2}^{\mathrm{II}}\left[n(K)^{2}-r^{2}-\sum_{H \supset K}^{\mathrm{II}}\left(n(H)^{2}-r^{2}\right)\right] .
\end{aligned}
$$

$$
\begin{aligned}
& k^{2}-\sum_{H, 1}^{1} k(H)^{2}=\sum_{K, 2}^{1}\left[k(K)^{2}-\sum_{H \supset K}^{1} k(H)^{2}\right] \\
& \text { where } k=\alpha_{3}-\alpha_{1}-\alpha_{2}+1 .
\end{aligned}
$$

$$
n k-\sum_{H, 1}^{\mathbb{I}} n(H) k(H)=\sum_{K, 2}^{\mathbb{I}}\left[n(K) k(K)-\sum_{H \supset K}^{I} n(H) k(H)\right] .
$$

Squaring the Borel formula gives

$$
\begin{aligned}
n^{2}-r^{2}= & \sum_{i=1}^{p}\left(n\left(H_{i}\right)^{2}-r^{2}\right) \\
& +2 \sum_{i=1}^{p} \sum_{\substack{j=1 \\
i<j}}^{p}\left[n\left(H_{i}\right) n\left(H_{j}\right)-r\left\{n\left(H_{i}\right)+n\left(H_{j}\right)\right\}+r^{2}\right],
\end{aligned}
$$

where $H_{1}, \cdots, H_{p}$ are the corank one subtori, which belong to $f$.

Equality (1) follows, since each pair $\left(H_{i}, H_{j}\right), i<j$, appears exactly once on the right-hand side, associated with $K=\left(H_{i} \cap H_{j}\right)^{\circ}$.

Equality (2) follows similarly by squaring the formula in Theorem 2.5(ii), and equality (3) follows by multiplying the Borel formula by the formula of Theorem 2.5(ii).

Theorem 3.1(i) is now established, for it is quite clear that nothing is added to the formula when the summations are allowed to range in the way stated. 
Theorem 3.1(ii) differs from (i) only by the Borel formula and equality (1), above, and so it follows easily.

\section{REFERENCES}

1. C. J. Allday, A note on a theorem of W.-Y. Hsiang, University of Hawaii, 1972 (preprint).

2. C. J. Allday and T. Skjelbred, The Borel formula and the topological splitting principle for torus actions on a Poincaré duality space, Ann. of Math. (2) 100 (1974), 322-325.

3. A. Borel et al., Seminar on transformation groups, Ann. of Math. Studies, no. 46, Princeton Univ. Press, Princeton, N. J., 1960. MR 22 \#129.

4. N. Bourbaki, Éléments de mathématique. Fasc. XXVII. Algébre commutative, Actualités Sci. Indust., no. 1290, Hermann, Paris, 1961. MR 36 \#146.

5. G. E. Bredon, Fixed point sets of actions on Poincaré duality spaces, Topology 12 (1973), 159-175.

6. - Homotopical properties of fixed point sets of circle group actions. I, Amer. J. Math. 91 (1969), 874-888. MR 41 \#4534.

7. - Introduction to compact transformation groups, Academic Press, New York, 1972.

8. T. Chang and T. Skjelbred, Group actions on Poincaré duality spaces, Bull. Amer. Math. Soc. 78 (1972), 1024-1026. MR 46 \#6346.

9. - The topological Schur lemma and related results, Ann. of Math. (2) 100 (1974), 307-321.

10. D. Golber, Torus actions on a product of two odd spheres, Topology 10 (1971), 313-326. MR 44 \#1024.

11. W.-Y. Hsiang, On generalizations of a theorem of A. Borel and their applications in the study of topological actions, Topology of Manifolds, Markham, Chicago, Ill., 1970.

12. - On some fundamental theorems in cohomology theory of topological transformation groups, Taita J. Math. 2 (1970), 61-87. MR 46 \#8247; A summary of these results may be found in: Some fundamental theorems in cohomology theory of topological transformation groups, Bull. Amer. Math. Soc. 77 (1971), 1094-1098. MR 46 \#8249.

13. S. Mac Lane, Homology, Die Grundlehren der math. Wissenschaften, Band 1.14, Academic Press, New York; Springer-Verlag, Berlin, 1963. MR 28 \#122.

14. D. Quillen, The spectrum of an equivariant cohomology ring. I, Ann. of Math. (2) 94 (1971), 549-572. MR 45 \#7743.

15. J.-P. Serre, Algèbre locale. Multiplicités, 2ième éd., Lecture Notes in Math., no. 11, Springer-Verlag, Berlin and New York, 1965. MR 34 \#1352.

16. T. Skjelbred, Thesis, University of California, Berkeley, Calif., 1972.

DEPARTMENT OF MATHEMATICS, UNIVERSITY OF HAWAII, HONOLULU, HAWAII 96822 